\title{
Title: An artificial impact on the asteroid 162173 Ryugu formed a crater in the gravity-dominated regime
}

Authors: M. Arakawa ${ }^{*}$, T. Saiki², K. Wada ${ }^{3}$, K. Ogawa ${ }^{21,1}$ T. Kadono ${ }^{4}$, K. Shirai ${ }^{2,1}$, H. Sawada $^{2}$, K. Ishibashi ${ }^{3}$, R. Honda ${ }^{5}$, N. Sakatani ${ }^{2}$, Y. Iijima ${ }^{2 \S}$, C. Okamoto ${ }^{1 \S}$, H. Yano ${ }^{2}$, Y.

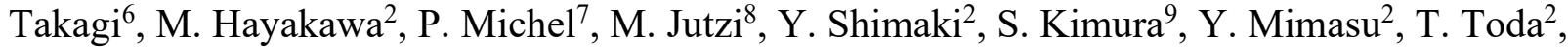
H. Imamura ${ }^{2}$, S. Nakazawa ${ }^{2}$, H. Hayakawa ${ }^{2}$, S. Sugita ${ }^{10,3}$, T. Morota ${ }^{10}$, S. Kameda ${ }^{11}$, E. Tatsumi $^{20,12,10}$, Y. Cho ${ }^{10}$, K. Yoshioka ${ }^{10}$, Y. Yokota ${ }^{2,5}$, M. Matsuoka ${ }^{2}$, M. Yamada ${ }^{3}$, T. Kouyama $^{13}$, C. Honda ${ }^{14}$, Y. Tsuda ${ }^{2}$, S. Watanabe ${ }^{15,2}$, M. Yoshikawa ${ }^{2,19}$, S. Tanaka ${ }^{2,19}$, F. Terui ${ }^{2}$, S. Kikuchi ${ }^{2}$, T. Yamaguchi ${ }^{2 \dagger}$, N. Ogawa ${ }^{2}$, G. Ono ${ }^{16}$, K. Yoshikawa ${ }^{16}$, T. Takahashi ${ }^{2 \ddagger}$, Y. Takei $^{16,2}$, A. Fujii ${ }^{2}$, H. Takeuchi ${ }^{2,19}$, Y. Yamamoto ${ }^{2,19}$, T. Okada ${ }^{2,10}$, C. Hirose ${ }^{16}$, S. Hosoda ${ }^{2}$, O. Mori $^{2}$, T. Shimada 2 , S. Soldini ${ }^{17}$, R. Tsukizaki ${ }^{2}$, T. Iwata ${ }^{2}$, M. Ozaki ${ }^{2,19}$, M. Abe ${ }^{2,19}$, N. Namiki $^{18,19}$, K. Kitazato ${ }^{14}$, S. Tachibana ${ }^{10}$, H. Ikeda ${ }^{16}$, N. Hirata ${ }^{14}$, N. Hirata ${ }^{1}$, R. Noguchi ${ }^{2}$, A. Miura $^{2}$.

\section{Affiliations:}

15 1. Kobe University, Kobe 657-8501, Japan.

2. Institute of Space and Astronautical Science, Japan Aerospace Exploration Agency, Sagamihara 252-5210, Japan.

3. Planetary Exploration Research Center, Chiba Institute of Technology, Narashino 275-0016, Japan.

4. University of Occupational and Environmental Health, Kitakyusyu 807-8555, Japan.

5. Kochi University, Kochi 780-8520, Japan.

6. Aichi Toho University, Nagoya 465-8515, Japan.

7. The Côte d'Azur Observatory, 06304 Nice Cedex 4, France.

8. Physics Institute, University of Bern, NCCR PlanetS, Gesellschaftsstrasse 6, 3012, Bern, Switzerland.

9. Tokyo University of Science, Noda 278-8510, Japan.

10. The University of Tokyo, Tokyo 113-0033, Japan.

11. Rikkyo University, Tokyo 171-8501, Japan.

12. University of La Laguna, 38205 San Cristóbal de La Laguna, Spain.

13. National Institute of Advanced Industrial Science and Technology, Tokyo 135-0064, Japan.

14. The University of Aizu, Aizu-Wakamatsu 965-8580, Japan.

15. Nagoya University, Nagoya 464-8601, Japan.

16. Research and Development Directorate, Japan Aerospace Exploration Agency, Sagamihara 252-5210, Japan.

17. University of Liverpool, Liverpool L3 5TQ, United Kingdom.

18. National Astronomical Observatory of Japan, Mitaka 181-8588, Japan.

19. SOKENDAI (The Graduate University for Advanced Studies), Hayama 240-0193, Japan.

20. Instituto de Astrofísica de Canarias, La Laguna, Tenerife, E38205, Spain.

21. JAXA Space Exploration Center, Japan Aerospace Exploration Agency, Sagamihara 2525210, Japan.

${ }^{*}$ Corresponding author: E-mail: masahiko.arakawa@penguin.kobe-u.ac.jp

${ }^{\dagger}$ Current affiliation: Mitsubishi Electric Corporation, Kamakura 247-8520, Japan.

Current affiliation: NEC Corporation, 1-10 Nisshin-cho, Fuchu, Tokyo 183-0036, Japan.

${ }^{\S}$ Deceased. 
Abstract: The Hayabusa2 spacecraft investigated the small asteroid Ryugu, which has a rubble pile structure. We describe an impact experiment on Ryugu using Hayabusa2's Small Carry-on Impactor (SCI). The impact produced an artificial crater with a diameter $>10$ meters, which has a semicircular shape, an elevated rim and a central pit. Images of the impact and resulting ejecta were recorded by the Deployable CAMera 3 (DCAM3) for $>8$ minutes, showing the growth of an ejecta curtain and deposition of ejecta onto the surface. The ejecta curtain, outer edge of ejecta, was asymmetric, heterogeneous, and never fully detached from the surface. The artificial crater was formed in the gravity-dominated regime: the gravity limited the crater growth; we discuss the implication of this observation for Ryugu's surface age.

Main Text: The Hayabusa2 spacecraft reached the near-Earth asteroid (162173) Ryugu in June 2018, showing that this small asteroid is covered with boulders with sizes up to $\sim 160 \mathrm{~m}(1,2)$. The surface is covered with a regolith layer composed of boulders and granules under microgravity conditions (gravitational acceleration about $1 \times 10^{-4} \mathrm{~m} \mathrm{~s}^{-2}$ ), which was expected to have strength originating from cohesion forces between regolith grains, with the maximum theoretical strength of the surface layer predicted to be $1 \mathrm{kPa}(3)$. Impact craters formed under those conditions should be limited in size by this surface strength, which reduces the expected crater diameter compared to a strengthless surface (4). Crater sizes produced by hypervelocity impacts on small bodies can be predicted using a variety of crater scaling laws. The number and sizes of craters on asteroids (including Ryugu) can be used to date their surfaces, but different crater scaling laws give ages that differ by more than an order of magnitude (1).

Hayabusa2 spacecraft was equipped with a Small Carry-on Impactor (SCI) designed to launch a $2 \mathrm{~kg}$ copper projectile at a velocity of $2 \mathrm{~km} \mathrm{~s}^{-1}$ to form an artificial impact crater (referred to hereafter as the SCI crater) on the surface of Ryugu. The SCI crater was intended to expose the asteroid subsurface for remote sensing and sample collection $(5,6)$. The Deep Impact spacecraft performed an impact experiment on a small body and formed an artificial impact crater on the nucleus of the comet 9P/Tempel 1(7-9). The SCI impact can be used to test crater scaling laws, for the crater size and ejecta velocity distribution, which were constructed by laboratory experiments (10-12), and be compared with numerical simulations of the impact cratering processes in a microgravity environment (13).

The SCI impact operation was carried out on 5 April 2019 (14). The impact and evolution of impact ejecta were simultaneously observed by Hayabusa2's Deployable CAMera 3 (DCAM3) (14). About 3 weeks after the SCI impact, Hayabusa2 searched for evidence of the impact crater using its Optical Navigation Camera-Telescopic (ONC-T) from an altitude of $1.7 \mathrm{~km}$ with a resolution of $\sim 18 \mathrm{~cm} /$ pixel. The fresh crater was identified at latitude $7.9^{\circ} \mathrm{N}$ and longitude $301.3^{\circ} \mathrm{E}$ (Figure 1A-B), $\sim 20 \mathrm{~m}$ from the aiming point (which was latitude $6.00^{\circ} \mathrm{N}$, longitude $303.00^{\circ} \mathrm{E}$ ). This location is in a northern part of Ryugu's equatorial ridge (2). The impact angle was estimated to be $\sim 60^{\circ}$ from the local horizontal surface (14).

Comparison of ONC-T images taken from the same altitude before and after the impact (Fig. 1A-B) shows the formation of an artificial impact crater. A dozen boulders with sizes of at least tens of $\mathrm{cm}$ were driven away from the surface, and a large block with a size of $5 \mathrm{~m}$ (which we refer to as the Mobile Block, $\mathrm{MB}$ ) was excavated and moved $\sim 3 \mathrm{~m}$ northwest and $>1 \mathrm{~m}$ above the initial surface by the impact. Before the impact, $\mathrm{MB}$ was adjacent to another large block (Stable Block, SB) (Fig. 1A), but SB was hardly moved by the SCI impact. SB might be part of a larger block buried below the crater floor. We speculate that SB and its subsurface extension 45 halted the crater growth toward the southeast. Alternatively, SCI may have impacted closer to $\mathrm{MB}$ than $\mathrm{SB}$, allowing the higher pressure to move MB but not SB. 
To investigate the crater morphology profile, we generated a Digital Elevation Map (DEM) (Fig. 2A) from ONC-T images (14). This shows an elevated rim with a height of $\sim 40 \mathrm{~cm}$ surrounding the SCI crater (Fig. 2B). The elevated rim could be composed of ejecta deposits and/or structural uplifts. We used the DEM to produce an ortho-corrected (a geometrically accurate) image on which we traced the top of the rim to determine the crater shape (Fig. 1C). The SCI crater is semi-circular with a rim to rim diameter $D_{\text {rim }}$ of $17.6 \pm 0.7 \mathrm{~m}$. This shape is not due to the oblique impact but the effect of a large block (possibly SB) southeast of the impact point (Fig. 1B) (14). Impact craters are usually symmetrical at this impact angle, so we assume that the SCI impact point is the center of the semicircular crater rim. A crater's apparent diameter $D$ is conventionally measured at an initial surface elevation. Using the DEM, we determine the SCI crater to have $D=14.5 \pm 0.8 \mathrm{~m}$ (Fig. 2A).

There is a small pit close to the impact point on the crater floor. The pit entrance is at a depth of $1.7 \mathrm{~m}$ from the initial surface (Fig. 2B); the diameter of the pit is $\sim 3 \mathrm{~m}$ and its depth is $0.6 \mathrm{~m}$. The pit has a conical shape similar to the simple craters formed in laboratory experiments (10, 15). Central pits are commonly observed in craters smaller than a few hundred meters on the Moon because the lunar subsurface has a layered structure consisting of a relatively hard layer covered with softer cohesionless regolith (16). Therefore, the pit could be explained if the SCI impact occurred above a subsurface layer with a cohesional strength. Some small natural craters on Ryugu also have pit features (fig. S4). The cohesional strength of this putative subsurface layer would be between 140 and $670 \mathrm{~Pa}$ (14). Alternatively, a central pit could be formed on compressive material if the projectile density is larger than that of the targets $(7,8)$. Because the pit depth is $2.7 \mathrm{~m}$ from the rim top, the depth to rim diameter ratio of the SCI crater is $0.15 \pm$ 0.01 . This value is consistent with the natural craters found on Ryugu, which range from 0.14 to $0.20(1)$.

Several boulders with sizes of 0.6 to $1.9 \mathrm{~m}$ are present within the crater. The crater wall appears to be smooth (Fig. 1D) because the boulders are buried inside the wall. They could have been exposed by the excavation flow of subsurface materials during the crater growth. The sizefrequency distribution (fig. S5) of boulders embedded in the wall is about one third of that on a nearby area of undisturbed surface (the $\mathrm{C} 01-\mathrm{C}$ region at $10^{\circ} \mathrm{N}, 300^{\circ} \mathrm{E}$, about $20 \mathrm{~m}$ northwest of the impact point), where the size-frequency distribution in the undisturbed area could be similar to the impact point before the SCI impact in submeter range. The SCI crater wall must be composed primarily of regolith with rock sizes smaller than $20 \mathrm{~cm}$. Assuming that the crater wall represents the subsurface structure, we conclude that the subsurface layer is dominated by regolith with rock sizes smaller than $20 \mathrm{~cm}$.

35 DCAM3 observed the impact ejecta from the moment of impact until more than 8 minutes later. Figure 3A-F shows the images taken by DCAM3 from $5 \mathrm{~s}$ to $498 \mathrm{~s}$ after the impact. The image at $5 \mathrm{~s}$ shows the impact ejecta curtain growing northwards from the surface at an angle of $40^{\circ}$ (Fig. 3A, fig. S6A). Subsequent images show the impact ejecta curtain was asymmetric and contained multiple rays in the early stages. Ejecta growth toward the south is not observed, but low speed ejecta at the base of the ejecta curtain are visible in faint scattered light.

At $36 \mathrm{~s}$, the northward ejecta ray becomes clearer and more extensive. Two more ejecta rays appear at this time (Fig. 3B, fig. S6B). Multiple rays of ejecta were previously observed in the Deep Impact experiments (7). A continuous portion of ejecta curtain, which appears as a shadow at the base of the ejecta curtain, begins to grow vertically and horizontally. This growth could originate from the expanding crater due to ongoing excavation of the subsurface material. 
These three ejecta rays continue to grow in both size and thickness, becoming more visible at $100 \mathrm{~s}$ and $192 \mathrm{~s}$ (Fig. 3C-D; fig. S6C-D), indicating that the crater growth is continuing at these times. An additional ejecta ray appears as a dark shadow in these images at the center of the ejected region, so we interpret it as moving toward the line of sight of DCAM3, but there is no visible ejecta curtain in the southeast area. The surface of Ryugu is covered with many large boulders with a power law size distribution (1), which we presume also applies to boulders buried in the subsurface. The SCI impact area was covered with boulders $>0.6 \mathrm{~m}$, some of which had most of their volume buried in the ground (Fig. 1A). These boulders could cause a heterogeneous ejecta growth, which would then result in ejecta rays, i.e. the boulders on or under the surface might stop the excavation flow, separating the ejecta curtain into several rays. The ejecta curtain formed by the Deep Impact collision exhibited an up-range zone of avoidance (ZoA) (7). We see a similar ZoA for the SCI impact, which may be caused by the oblique nature of the impact, as in Deep Impact. However, the resulting semicircular shape of the SCI crater demonstrates the influence of large boulders.

We analyzed the distribution of ejecta rays around the SCI crater using the image at $192 \mathrm{~s}$ (Fig. 4A) together with ONC-T images of the SCI crater area obtained before the impact. The direction and covered surface area of each ejecta ray were identified on the images taken by ONC-T with reference to the DCAM3 line of sight. Fig. 4B shows the distribution of the main ejecta rays mapped on an ONC-T surface image; each numbered sector on the map corresponds to the numbered separated ejecta ray in Fig. 4A, while the continuous ejecta curtain corresponds with a semicircle at the center. The whole ejecta, including the rays as well as the continuous curtain, was only distributed on the northwest-side. This distribution map can be compared with an ONC-T $v$-band $(0.55 \mu \mathrm{m})$ reflectance difference map shown in Fig. $4 \mathrm{C}(14)$ : we interpret regions with larger decrease of the reflectance factor as being covered with a thicker ejecta deposit. These regions are arranged asymmetrically around the crater: there are 4 discrete extended areas and none (or only a very faint one) to the southeast of the crater. These features in the reflectance difference map are consistent with the ejecta distribution map, so we are convinced that these maps represent the spatial distribution of the ejecta deposit and the ejected subsurface material has a lower reflectance than the average Ryugu surface.

At $396 \mathrm{~s}$ and $498 \mathrm{~s}$, the surface of Ryugu is visible through the ejecta curtain (Fig. 3, E and F; fig. S6, E and F), indicating that the optical thickness of the ejecta curtain was lower than at 192 s. A deep gray elliptical area appears, surrounded by the ejecta rays. We interpret this as the intrinsic color of the surface, and suggest that it represents the excavated area and/or the ejecta

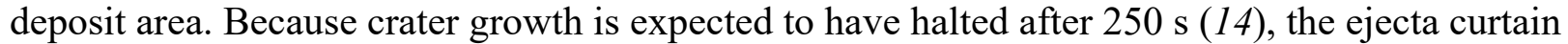
observed in these images must have been falling towards the surface on a ballistic trajectory. The major axis of the ellipse at $498 \mathrm{~s}$ was determined to be $36 \mathrm{~m} \mathrm{(14).}$

Detachment of the whole ejecta curtain from the ground is generally expected during crater formation in the strength-dominated regime but not in the gravity-dominated regime (5). No detachment of the ejecta curtain was observed for the SCI crater formation, including in the final observation at $498 \mathrm{~s}$ (Fig. 3A-F). In Fig. 3 and Movie S2, the ejecta curtain appears to be deposited at the crater rim. This is supported by the ONC-T reflectance difference map in Fig. $4 \mathrm{C}$, so the elevated rim surrounding the SCI crater could be (at least partially) composed of the ejecta deposits. This is expected for crater formation in the gravity-dominated regime $(15,17)$. We conclude that the structures of the SCI crater and the ejecta curtain indicate that they formed in the gravity-dominated regime. 
For the crater formation to be dominated by gravity, the effective strength of the target must be less than 1.3 $\mathrm{Pa}(14)$. Cohesionless materials such as sand can satisfy that small strength at the depth shallower than $10 \mathrm{~m}$, because the sub-surface shear strength originates from the frictional stress exerted on cohesionless materials and this stress is proportional to the surface gravity. We calculate the expected SCI crater radius using the conventional scaling law in the gravitydominated regime: $\pi_{R}=k_{1} \cdot \pi_{2}{ }^{-\mu /(2+\mu)} \pi_{4}{ }^{(2+\mu-6 v) /(3(2+\mu))} \quad(18)$, where $\pi_{R}=R(\rho / m)^{1 / 3}$, $\pi_{2}=\left(g a / u^{2}\right), \pi_{4}=(\rho / \delta)$, and $R, \rho, m, a, u, \delta$ are the apparent crater radius, the target density, the projectile mass, the projectile radius, the impact velocity, and the projectile density, respectively, and $k_{1}, \mu$, and $v$ are empirical parameters determined by laboratory experiments, which applies to typical cohesionless surfaces such as coarse or loose sand (14). The calculated expected radius is from $6.9 \mathrm{~m}$ to $7.7 \mathrm{~m}$, which is close to the apparent radius of the observed SCI crater of $7.3 \pm 0.4 \mathrm{~m}$. We conclude the SCI crater formed on a cohesionless surface and that the broader surface of Ryugu must be composed of sand-like cohesionless material. According to the conventional scaling law, we can estimate the optimal $k_{1}$ value applicable to the surface of Ryugu from the SCI crater radius $R=7.3 \mathrm{~m}(14)$; we obtain $k_{1}=0.62$ if we assume that other parameters have typical values for sand, and can be extrapolated to cohesionless targets at high impact velocities: $\mu=0.41$ and $v=0.4(14,18)$. We derive the crater size scaling law for the surface of Ryugu; $\pi_{R}=0.62 \cdot \pi_{2}{ }^{-0.17} \pi_{4}{ }^{0.0014}$.

Our results affect the interpretation of the crater chronology on Ryugu. Based on collision frequency models for the asteroid Main Belt $(19,20)$, two estimates of the surface age of Ryugu have been obtained: $(1.58 \pm 0.47) \times 10^{8}$ years for a surface composed of dry-soils with cohesion or $(8.9 \pm 2.5) \times 10^{6}$ years for a cohesionless surface $(1)$. Our conclusion that the SCI crater was formed in the gravity-dominated regime on a cohesionless surface supports the younger age estimate. It also suggests that the crater retention age of the top $1 \mathrm{~m}$ of Ryugu's surface is $\sim 1 \times 10^{5}$ years or younger, assuming crater production functions for near-Earth orbits $(14,19,20)$.

\section{References and Notes:}

1. S. Sugita et al., The geomorphology, color, and thermal properties of Ryugu: Implications for parent-body processes. Science 364, eaaw0422 (2019). doi: 10.1126/science.aaw0422

2. S. Watanabe et al., Hayabusa 2 arrives at the carbonaceous asteroid 162173 Ryugu-A spinning top-shaped rubble pile. Science 364, 268-272 (2019). doi: 10.1126/science.aav8032

3. K. Wada et al., Asteroid Ryugu before the Hayabusa2 encounter. Prog. Earth Planet. Sci. 5, 82 (2018). doi: 10.1186/s40645-018-0237-y

4. K. Holsapple, I. Giblin, K. Housen, A. Nakamura, E. Ryan, Asteroid impacts: Laboratory experiments and scaling laws. in Asteroids III, University of Arizona Press, Tucson, pp. 443462 (2002).

5. M. Arakawa et al., Scientific objectives of Small Carry-on Impactor (SCI) and Deployable Camera 3 Digital (DCAM3-D): observation of an ejecta curtain and a crater formed on the surface of Ryugu by an artificial high-velocity impact. Space Sci. Rev. 208, 187-212 (2017). doi: 10.1007/s11214-016-0290-z

6. S. Tachibana et al., Hayabusa2: Scientific importance of samples returned from C-type nearEarth asteroid (162173) 1999 JU3. Geochem. J. 48, 571-587 (2014). doi: 10.2343/geochemj.2.0350

7. P. H. Schultz, et al., The Deep Impact oblique impact cratering experiment. Icarus, 191, 84122 (2007). 10.1016/j.icarus.2007.06.031 
8. P. H. Schultz, C. M. Ernst, J. L. Anderson, Expectations for crater size and photometric evolution from the Deep Impact collision. Space Sci. Rev. 117, 207-239 (2005). doi: $10.1007 / \mathrm{s} 11214-005-3383-7$

9. M. F. A'hearn, et al., Deep impact: excavating comet Tempel 1. Science 310, 258-264 (2005). doi: 10.1126/science.1118923

10. K. Matsue, M. Yasui, M. Arakawa, S. Hasegawa, Measurements of seismic waves induced by high-velocity impacts: Implications for seismic shaking surrounding impact craters on asteroids. Icarus 338, 113520 (2020). doi:10.1016/j.icarus.2019.113520

11. E. Tatsumi, S. Sugita, Cratering efficiency on coarse-grain targets: Implications for the dynamical evolution of asteroid 25143 Itokawa. Icarus 300, 227-248 (2018). doi:10.1016/j.icarus.2017.09.004

12. M. J. Cintala, L. Berthoud, F. Hörz, Ejection-velocity distributions from impacts into coarsegrained sand. Meteorit. Planet. Sci. 34, 605-623 (1999). doi:10.1111/j.19455100.1999.tb01367.x

13. M. Jutzi, K. A. Holsapple, K. Wünneman, P. Michel, Modeling asteroid collisions and impact processes. in Asteroids IV, University of Arizona Press, Tucson, pp. 679-699 (2015). doi: 10.2458/azu_uapress_9780816532131-ch035

14. Materials and Methods are available as Supplementary Materials.

15. H. J. Melosh, Impact cratering: A geologic process. Oxford University Press, New York (1989).

16. Z. Yue et al., Lunar regolith thickness deduced from concentric craters in the CE-5 landing area. Icarus 329, 46-54 (2019). doi: 10.1016/j.icarus.2019.03.032

17. K. R. Housen, R. M. Schmidt, K. A. Holsapple, Crater ejecta scaling laws: Fundamental forms based on dimensional analysis. J. Geophys. Res. Solid Earth 88, 2485-2499 (1983). doi: 10.1029/JB088iB03p02485

18. K. R. Housen, K. A. Holsapple, Ejecta from impact craters. Icarus 211, 856-875 (2011). doi: 10.1016/j.icarus.2010.09.017

19. W. F. Bottke Jr. et al., The fossilized size distribution of the main asteroid belt. Icarus $\mathbf{1 7 5}$, 111-140 (2005). doi: 10.1016/ j.icarus.2004.10.026

20. D. P. O'Brien, R. Greenberg, The collisional and dynamical evolution of the main-belt and NEA size distributions. Icarus 178, 179-212 (2005). doi: 10.1016/j.icarus.2005.04.001

21. T. Saiki et al., The small carry-on impactor (SCI) and the Hayabusa2 impact experiment. Space Sci. Rev. 208, 165-186 (2017). doi: 10.1007/s11214-016-0297-5

22. K. Ogawa et al., System configuration and operation plan of Hayabusa2 DCAM3-D camera system for scientific observation during SCI impact experiment. Space Sci. Rev. 208, 125142 (2017). doi: 10.1007/s11214-017-0347-7

23. K. Ishibashi et al., Performance of Hayabusa2 DCAM3-D camera for short-range imaging of SCI and ejecta curtain generated from the artificial impact crater formed on asteroid 162137 Ryugu (1999JU3). Space Sci. Rev. 208, 213-238 (2017). doi: 10.1007/s11214-016-0298-4

24. D. E. Gault, J. A. Wedekind, Experimental studies of oblique impact. Proc. Lunar Planet. Sci. Conf. 9th, 3843-3875 (1978).

25. R. Szeliski, Computer vision: Algorithms and applications. Springer, London (2011). doi: 10.1007/978-1-84882-935-0

26. W. Schwanghart, D. Scherler, Short communication: TopoToolbox2-MATLAB-based software for topographic analysis and modeling in Earth surface sciences. Earth Surf. Dynam. 2, 1-7 (2014). doi: 10.5194/esurf-2-1-2014 
27. H. M. B. Al-Hashemi, O. S. B. Al-Amoudi, A review on the angle of repose of granular materials. Powder Technol. 330, 397-417 (2018). doi: 10.1016/j.powtec.2018.02.003

28. D. E. Maxwell, Simple $Z$ model of cratering, ejection, and the overturned flap. in Impact and explosion cratering: Planetary and terrestrial implications, Pergamon Press, New York, pp. 1003-1008 (1977).

29. B. Hermalyn, P. H. Schultz, M. Shirley, K. Ennico, A. Colaprete, Scouring the surface: Ejecta dynamics and the LCROSS impact event. Icarus 218, 654-665 (2012). doi:10.1016/j.icarus.2011.12.025

30. M. Yasui, E. Matsumoto, M. Arakawa, Experimental study on impact-induced seismic wave propagation through granular materials. Icarus 260, 320-331 (2015). doi:10.1016/j.icarus.2015.07.032

31. S. Tsujido, M. Arakawa, A. I. Suzuki, M. Yasui, Ejecta velocity distribution of impact craters formed on quartz sand: Effect of projectile density on crater scaling law. Icarus 262, 79-92 (2015). doi:10.1016/j.icarus.2015.08.035

32. M. Kiuchi, A. M. Nakamura, K. Wada, Experimental study on gravitational and atmospheric effects on crater size formed by low-velocity impacts into granular media. J. Geophys. Res. 124, 1379-1392 (2019). doi:10.1029/2018JE005628

33. O. S. Barnouin, R. T. Daly, M. J. Cintala, D. A. Crawford, Impacts into coarse-grained spheres at moderate impact velocities: Implications for cratering on asteroids and planets. Icarus 325, 67-83 (2019). doi:10.1016/j.icarus.2019.02.004

34. C. Güttler, N. Hirata, A. M. Nakamura, Cratering experiments on the self armoring of coarsegrained granular targets. Icarus 220, 1040-1049 (2012). doi:10.1016/j.icarus.2012.06.041

35. S. Takizawa, H. Katsuragi, Scaling laws for the oblique impact cratering on an inclined granular surface. Icarus 335, 113409 (2020). doi:10.1016/j.icarus.2019.113409

Acknowledgments: Hayabusa2 was developed and built under the leadership of Japan Aerospace Exploration Agency (JAXA), with contributions from the German Aerospace Center (DLR) and the Centre National d'Études Spatiales (CNES), and in collaboration with NASA, and other universities, institutes, and companies in Japan. SCI was developed by JAXA, Kobe University, Chiba Institute of Technology, University of Occupational and Environmental Health, Aichi Toho University, Kyushu Institute of Technology, Hosei University, and other universities, institutes, and companies in Japan. DCAM3 was developed by JAXA, Kobe University, Kochi University, Chiba Institute of Technology, Tokyo University of Science, University of Occupational and Environmental Health, and other universities, institutes, and companies in Japan. M.Ar. thanks Minami Yasui for help in conducting laboratory impact experiments. K.W. is grateful to Hiroshi Kimura for discussions on the observational possibility of ejecta curtains. Funding: M.Ar.,T.O., T.M., S.W., N.N., K.W., T.M., K.O. M.Ab., S.Ka., A.M., S.Su., K.Yoshio, and Y.C. have been supported by KAKENHI from the Japanese Society for Promotion of Science (JSPS) (Grant Nos. JP16H04041, JP17H06459, JP15K05273, JP19H01951, JP19H00719, JP16H04044, JP19K03955, JP19H00727, JP17KK0097, JP18K11610, JP17H01175, JP19K14778). This study was supported by JSPS Core-to-Core program "International Network of Planetary Sciences." P.M. acknowledges funding support from the French space agency CNES and from Academies of Excellence: Complex systems and Space, environment, risk, and resilience, part of the IDEX JEDI of the Universite Côte d'Azur.

Author contributions: M.Ar. coordinated coauthor contributions; led the SCI and DCAM3 data acquisition, analyses, and interpretations; and wrote the paper, with contributions from K.W., 
K.O., T.Ka., K.S., K.I., R.H., N.S., P.M., M.J., and Y.S. SCI operations: T.Sa., K.W., T.Ka., K.S., Y.I., C.O., H.Y., Y.Taka., M.H., H.Im., and N.H. (Aizu) DCAM3 data acquisitions and reductions: K.W., K.O., K.S., H.Sa., K.I., R.H., N.S., Y.I., S.Kim., Y.M., T.To., S.N., and H.H. ONC data aqcuisitions and reductions: H.Sa., R.H., N.S., M.H., S.Su., T.M., S.Ka., E.T., Y.C., K.Yoshio., Y.Yo., M.M., M.Ya., T.Ko., and C.Ho. Image analysis: K.W., K.O., T.Ka., K.S., K.I., R.H., N.S., and Y.S. Spacecraft operations: T.Sa., K.O., K.S., H.Sa., R.H., N.S., H.Y., M.H., Y.M., S.N., Y.Ts., S.W., M.Yo., S.Tan., F.T., S.Kik., T.Y., N.O., G.O., K.Yoshiz., T.Ta., Y.Take., A.F., H.T., Y.Ya., T.O., C.Hi., S.H., O.M., T.Sh., S.So., R.T., T.I., M.O., M.Ab., N.N., K.K., S.Tac., and H.Ik. Shape modeling: K.S., Y.S., N.H. (Aizu), N.H. (Kobe), R.N., and A.M. materials availability: All images, and input data used in this study are available at the JAXA Data Archives and Transmission System (DARTS) at www.darts.isas.jaxa.jp/pub/ hayabusa2/paper/Arakawa_2020/. Additional data from Hayabusa2 ONC and DCAM3 data will be made available through the JAXA Data Archives and Transmission System website (https://www.darts.isas.jaxa.jp/).

\section{Supplementary Materials:}

Materials and Methods

Figures S1-S11

Table S1

References (21-35)

Movies S1-S2 


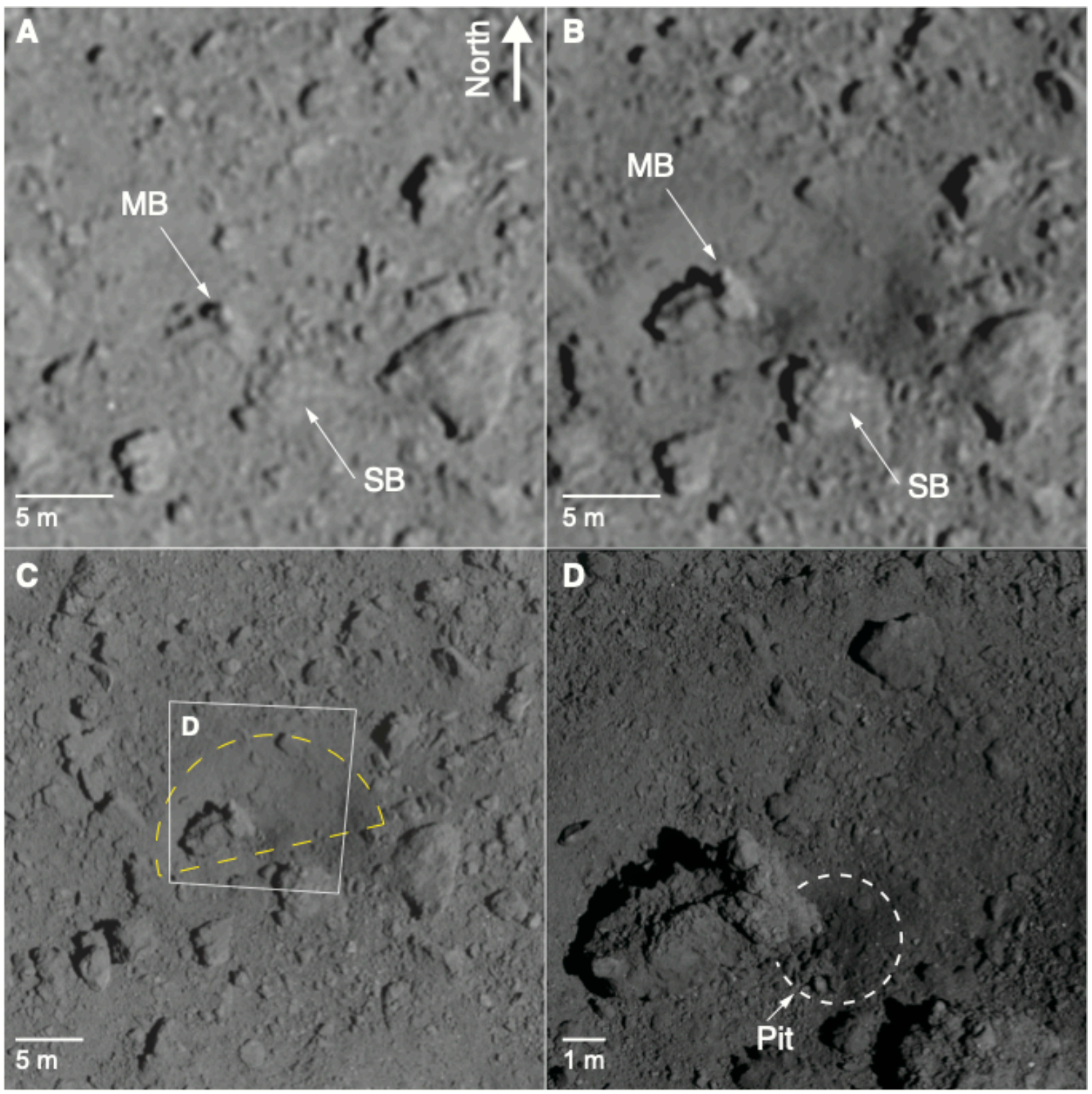

Fig. 1. ONC images of the SCI crater. (A) The area around the SCI aiming point before the impact, taken from $1.72 \mathrm{~km}$ altitude (image hyb2_onc_20190321_192706_tvf_12b. The Mobile Block (MB) and Stable Block (SB) are indicated with arrows. (B) The same location after the impact, observed from the same altitude (hyb2_onc_20190425_031226_tvf_12b). (C) Orthocorrected context image of the impact location (hyb2_onc_20190516_023615_tvf_12c) with the SCI crater shown by a yellow dashed semicircle. The white box indicates the region in panel D. (D) Close up image of the SCI crater taken from $115 \mathrm{~m}$ altitude (hyb2_onc_20190613_020217_tvf_12c). A small pit is shown by the dashed curve. For panels A and $\mathrm{B}$, the spatial resolution has been enhanced by a factor of four through deconvolution; the original images are shown in fig. S2. Movie S1 shows a blink comparison between panels A and B. 


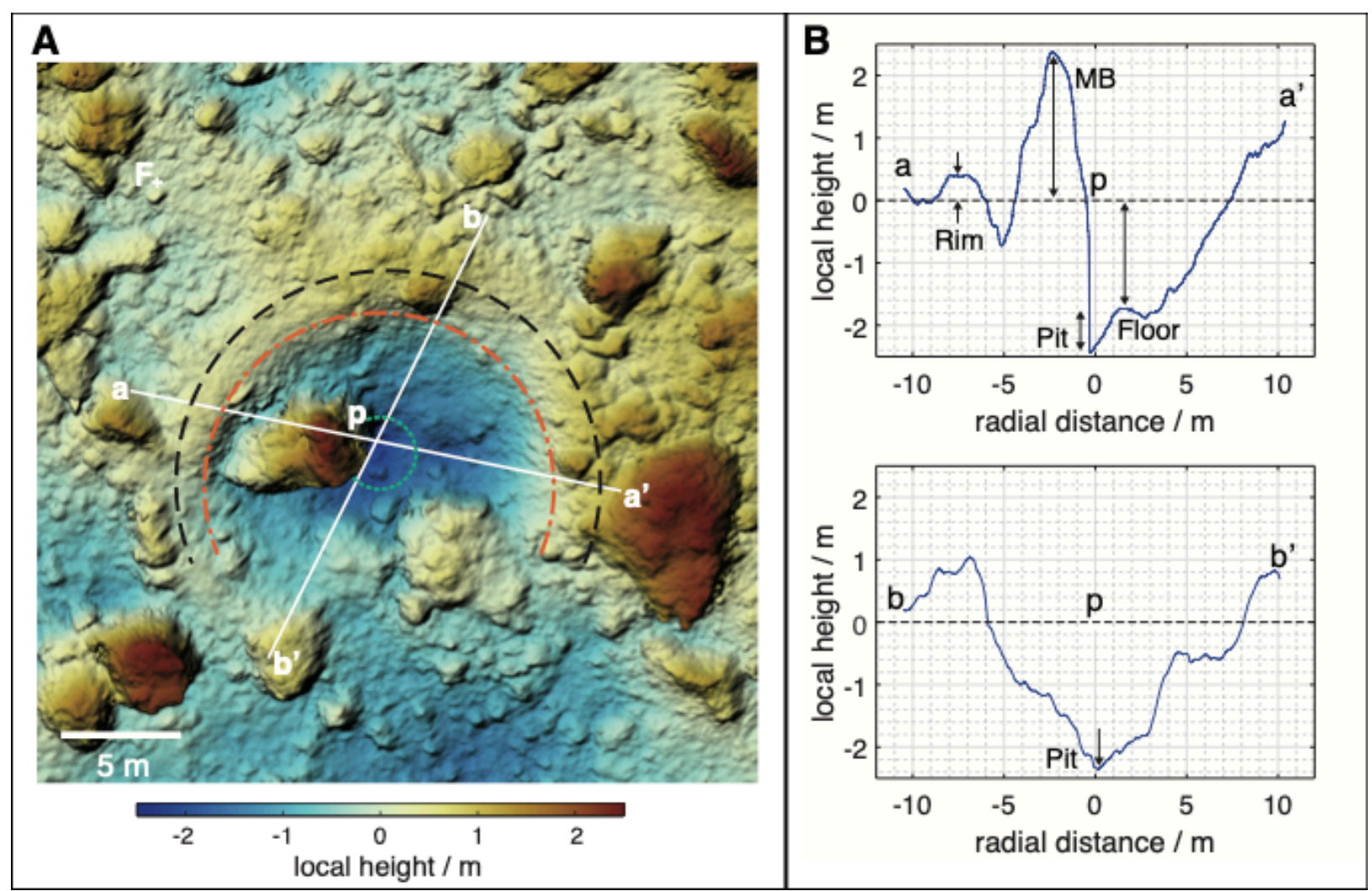

Fig. 2. Topography of the SCI crater. (A) Digital Elevation Map of the SCI impact area. The black dashed semicircle shows the crater rim with diameter $17.6 \mathrm{~m}$, and the red long dasheddotted semicircle shows the crater wall with diameter $14.5 \mathrm{~m}$. The green dotted circle shows the central pit. The local height is measured relative to point $\mathrm{F}$, which is labeled northwest of the crater. The white lines show the locations plotted in panel B. Figure S3 shows a contour map of this area. (B) Cross sections of the SCI crater. Radial distances are measured from the point $\mathrm{p}$. The rim, pit, crater floor, and MB are labelled. 


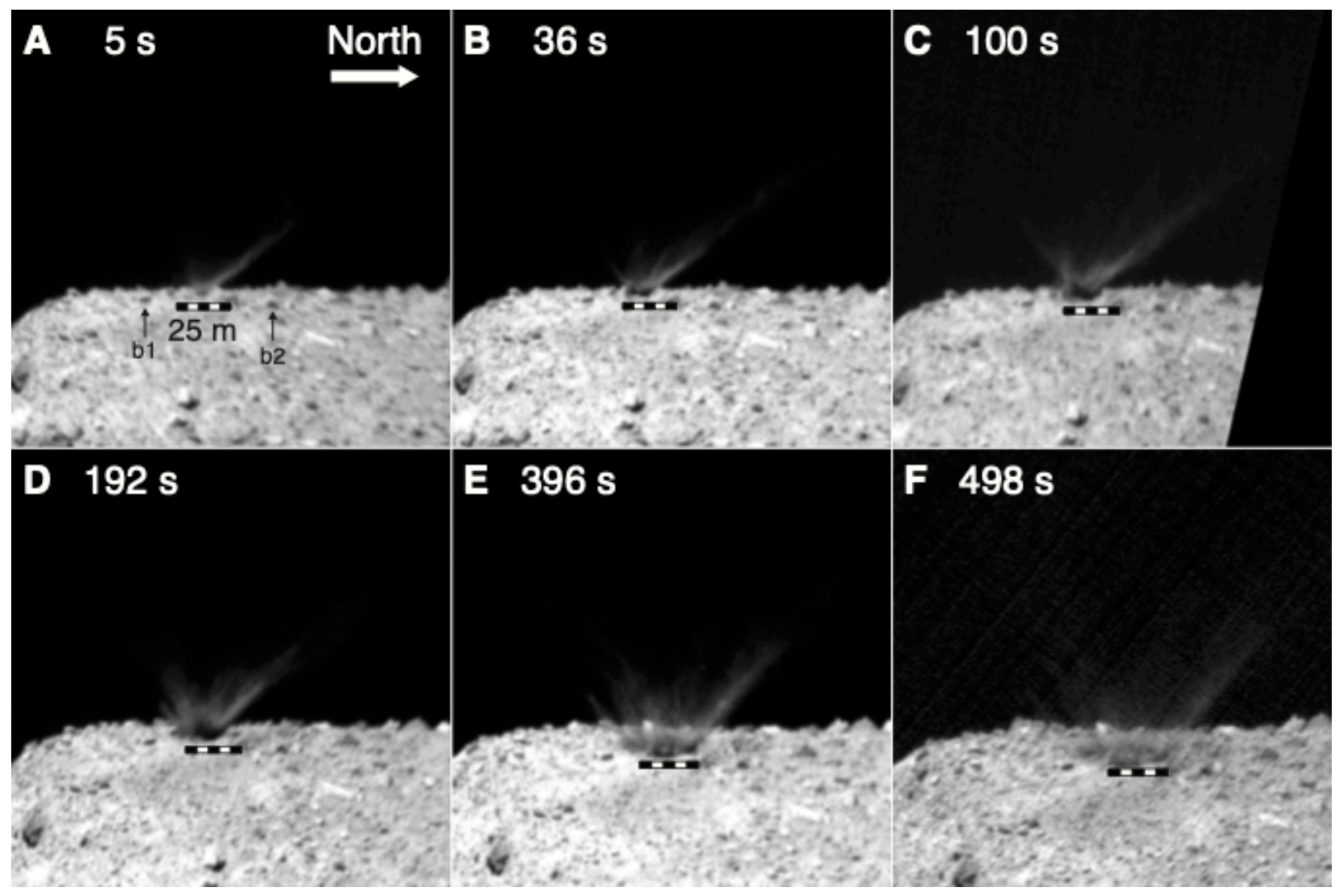

Fig. 3. DCAM3 images of the SCI impact. The images were taken at elapsed time after the impact of (A) 5 s (hyb2_dcam3d_img-f_01309_12a), (B) 36 s (hyb2_dcam3d_img-f_01340_12a),

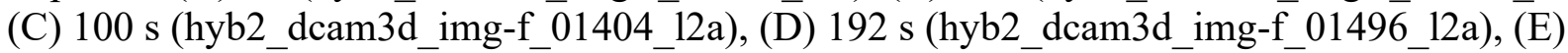
396 s (hyb2_dcam3d_img-f_01700_12a), and (F) 498 s (hyb2_dcam3d_img-f_01802_12a). The scale bars of $25 \mathrm{~m}$ were calibrated using the distance between the boulders labelled $\mathrm{b} \overline{1}$ and $\mathrm{b} 2$. Three visible rays are seen in a panel B and they are marked as 2, 3, and 4 in fig. S6B. The impact point moved towards DCAM3 during the observations, due to the rotation of Ryugu. Movie S2 shows an animated version of this figure. 

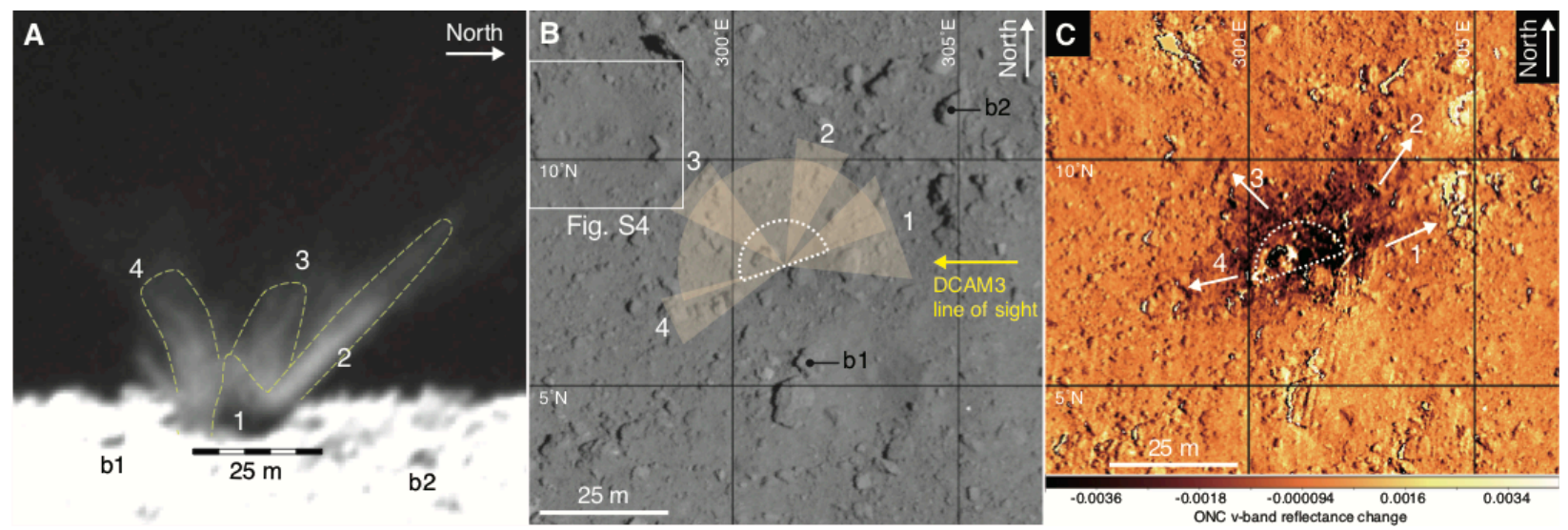

Fig. 4. Distribution of the SCI ejecta. (A) DCAM3 image taken at $192 \mathrm{~s}$, extracted from Figure 3D with increased contrast. Four ejecta rays are identified, enclosed with dashed lines, and numbered from 1 to 4 on the image. The scale bar and labelled boulder are the same as in Figure 3. (B) ONC $v$-band image map. The line of sight of DCAM3 at the time of Fig. 4A is shown with the yellow arrow. Numbered sectors correspond to the separate ejecta rays and a semicircle corresponds to the continuous ejecta curtain, both projected onto the ONC image and colored light orange. The white box indicates a natural pit crater northwest of the SCI crater, shown in detail in fig. S4. (C) Difference in ONC $v$-band reflectance between before and after the SCI impact. The typical reflectance factor of this area is about 0.018 , thus the darkest regions in this map have decreased in reflectance by about $20 \%$ after the impact. Four discrete extended darkened areas correspond with the ejecta rays and are numbered 1 to 4 . The same pre-impact shape model is used for both pre- and post-impact reflectance calculations (14), to emphasize weak reflectance changes due to ejecta deposit outside of the crater. 